\title{
Will the antimalarial drug take over to combat COVID-19?
}

\author{
Sivabakya T.K ${ }^{1}$ (D) - Srinivas $\mathrm{G}^{1}$
}

Received: 1 April 2020 / Accepted: 22 April 2020 / Published online: 29 April 2020

(C) Springer-Verlag GmbH Germany, part of Springer Nature 2020

\begin{abstract}
Background China has been fighting the epidemic of pneumonia-like diseases first detected for over a month in the city of Wuhan in December 2019. The disease epidemic is caused by a novel coronavirus, called COVID-19, which has now infected more than 700,000 people worldwide. With a death toll approaching that of China's SARS-CoV outbreak in 2002 and 2003, 2019-nCoV has contributed to an international emergency in public health, placing all health organizations on high alert. Such large numbers of infected and deceased people require an urgent need for reliable, inexpensive, and cheap drugs to control and reduce the outbreak.

Objective To systematically review and evaluate the pattern of COVID-19 and the treatment plans.

Methods This systematic review was conducted according to the Preferred Reporting Items for Systematic Reviews and MetaAnalyses (PRISMA) guidelines. The articles were searched from databases like PubMed, the Cochrane Library, ScienceDirect, and the Health Research and Development Information Network (HERDIN) combining MeSH and free-text terms.

Results This analysis highlights the agent of COVID-19 and the possible transmission. The current research taking place to overcome this complex disease and the urgent need to develop improved therapeutics are also discussed.

Conclusion Herein, we present an epidemiological overview of the currently available information on the treatment claimed to have helped to bring the situation under control.
\end{abstract}

Keywords Wuhan $\cdot$ China $\cdot$ COVID-19 $\cdot$ Outbreak $\cdot$ Chloroquine $\cdot$ Hydroxychloroquine

\section{Introduction}

Several cases of unknown etiology pneumonia have been confirmed and registered in Wuhan, Hubei Province, China since December 8, 2019 (Huang et al. 2020). Most of the sufferers worked or lived around the local Huanan Seafood Wholesale Market, where live animals were on sale. Extreme acute respiratory infection signs occurred in the early stages of this pneumonia, with some sufferers quickly developing acute respiratory distress syndrome (ARDS), severe breathing failure, and other significant complications. On January 7, 2020, a single coronavirus was detected from a patient's throat swab sample

Sivabakya T.K

sivasyd.nov7@yahoo.com

Srinivas $\mathrm{G}$

srinivas.g@tnmgrmu.ac.in

1 Department of Epidemiology, The Tamil Nadu Dr. M.G.R. Medical University, No. 69, Anna Salai, Guindy, Chennai, Tamil

Nadu 600032, India by the Chinese Center for Disease Control and Prevention (China CDC) and was eventually called COVID-19 by the World Health Organization (WHO) (Ji et al. 2020). This virus has now caused a total of 750,890 confirmed human infections worldwide, with 36,405 deaths (WHO Situation report 71, 31 March 2020 2020). The whole world looks forward to some kind of relief and treatment for this serious pandemic.

\section{Agent}

Coronaviruses belong to the Coronavirinae subfamily of the Coronaviridae family of the order Nidovirales, and this subfamily consists of four genera: Alphacoronavirus, Betacoronavirus, Gammacoronavirus, and Deltacoronavirus (Chen et al. 2020). Coronaviruses of zoonotic origin can, however, evolve into a strain capable of infecting mortally ill people ( $\mathrm{Su}$ et al. 2016).

COVID-19 is the seventh MERS-nCoV and SARS-nCoV coronavirus family member to infect humans. A genetic sequence of the virus was made available to the $\mathrm{WHO}$ via genome sequencing, which allowed various laboratories to 
produce diagnostic reverse transcription polymerase chain reaction (RT-PCR) tests specifically for viral RNA detection. COVID-19 is a category $2 \mathrm{~B} \beta \mathrm{CoV}$ with a genetic sequence similarity of more than $70 \%$ to SARS-nCoV (Hui et al. 2020). The origins of the new infection with coronavirus was ascertained as bats. With full genome sequences, Zhou and colleagues found that the virus is $96 \%$ the same as a bat coronavirus at the level of the entire genome (Zhou et al. 2020). $\mathrm{Wu}$ and colleagues performed a phylogenetic analysis of the entire viral genome and deduced that the virus was most closely associated with a group of previously isolated SARS-like coronaviruses from bats in China (Wu et al. 2020).

\section{Possible transmissions}

After the first cases of the COVID-19 disease were linked to direct access to Wuhan's Huanan Seafood Wholesale Market, it was suspected that the main mechanism was a transmission from animal to human. The cycle of adaptation was not associated with subsequent incidents, however. It was also known that the virus could also be transmitted from human to human and that the most frequent cause of COVID-19 spread is symptomatic individuals. The probability of transmission appears unlikely before symptoms arise, but it cannot be ruled out. There are also indications that the virus can be transmitted by people who remain asymptomatic.

Based on data from the first cases in Wuhan and inquiries performed by the Chinese CDC and local CDCs, the incubation period could typically be between 3 to 7 days and up to 2 weeks, as the longest duration from infection to symptoms was 12.5 days [95\% confidence interval (CI), 9.2 to 18 ] ( $\mathrm{Li}$ et al. 2020).

These data also showed that this novel outbreak doubled every 7 days, although the simple number of reproductions $\left(R_{0}\right)$ was 2.2. In other words, every patient transmits the infection to an additional 2.2 individuals on average. Of note, estimates for the SARS-CoV outbreak $R_{0}$ were about 3 in 2002-2003 (Bauch et al. 2005)

\section{Treatment approaches so far}

COVID-19 is not approved for specific antiviral therapy and does not have a vaccine. The condition is symptomatic, and the main treatment intervention for severely compromised patients is oxygen therapy. Mechanical ventilation may be needed in cases of respiratory failure refractory to oxygen therapy, while hemodynamic support is necessary for septic shock control.

On January 28, 2020, the WHO issued a paper summarizing guidelines from the $\mathrm{WHO}$ and clinical evidence collected from the treatment of previous $\mathrm{HCoV}$ epidemics. This study addresses measures to classify and sort patients with extreme acute respiratory disease, methods to avoid and contain infections, early intervention and monitoring therapy, guidelines for laboratory diagnosis, management of respiratory failure and ARDS, management of septic shock, prevention of complications, treatments, and consequences for pregnant patients (Cascella et al. 2020).

\section{The launch of a megatrial}

A combination of the drugs used against HIV, a malaria vaccine first tested during the Second World War, and a new antiviral against Ebola last year whose promise waned. On March 20, 2020, the WHO initiated a large global project, called SOLIDARITY, to find out if any of these drugs can be used with the new coronavirus infections to cure the deadly respiratory illness. The project also examines unapproved medications that have performed well with the other two deadly coronaviruses in animal studies, causing severe acute respiratory syndrome (SARS) and Middle East respiratory syndrome (MERS) (Kupferschmidt and Cohen 2020).

\section{Will the approved or investigational drug take over?}

There are no medications specifically licensed by the US Food and Drug Administration (FDA) for the treatment of COVID19 patients. In several hundred clinical trials that are ongoing across the globe, many drugs approved for other indications as well as other investigational drugs are being studied. Two of the approved drugs (chloroquine and hydroxychloroquine) and one of the commonly used research agents (remdesivir) are in the limelight for the whole world on that basis (Centers for Disease Control and Prevention, CDC 2020)

\section{Remdesivir}

Remdesivir is a large-scale intravenous antiviral investigative drug that inhibits viral replication by premature termination of RNA transcription and has in vitro activity against SARSCoV-2 and in vitro and in vivo activity against related beta coronaviruses (Mulangu et al. 2019).

Recently, remdesivir was recognized as a promising antiviral drug against a large array of RNA viruses. This is equivalent to adenosine, which integrates into the chains of embryonic viral RNA and results in premature termination. Warren et al. demonstrated that the non-human primate model's intravenous administration of $10 \mathrm{mg} / \mathrm{kg}$ of remdesivir resulted in concomitant persistent levels of its active form in the blood 
$(10 \mu \mathrm{M})$ and offered $100 \%$ protection against the most feared Ebola virus infection (Warren et al. 2016).

In an in vitro analysis, he time-of-addition assay showed that remdesivir functioned at a post-virus stage entry, which is following its putative antiviral mechanism as an analogue nucleotide (Wang et al. 2020).

\section{Chloroquine}

Chloroquine, a widely used drug for antimalarial and autoimmune disorders, has been recently described as a possible broad-spectrum antiviral medicine. Chloroquine is known to prevent viral infection by growing the endosomal $\mathrm{pH}$ needed for virus/cell fusion, as well as interfering with SARS-CoV cell receptor glycosylation (Vincent et al. 2005).

In addition to its antiviral function, chloroquine has an immune-modulating role that can improve synergistically its antiviral effect in vivo. Upon oral administration, chloroquine is spread extensively throughout the entire body, including the lung. The EC90 value of chloroquine in Vero E6 cells against COVID-19 was $6.90 \mu \mathrm{M}$, which can be clinically attainable as seen in the plasma of patients receiving $500 \mathrm{mg}$ of rheumatoid arthritis. The time-of-addition assay demonstrated, in an in vitro analysis, that chloroquine performed at both entry and post-entry stages of COVID-19 infections in Vero E6 cells (Wang et al. 2020).

\section{Hydroxychloroquine}

Hydroxychloroquine and chloroquine are cellular autophagy modulators that interact with enveloped viruses, such as retroviruses, flaviviruses, and coronaviruses, at late stages of replication (Savarino et al. 2003; Byrd and Horwitz 1991). Hydroxychloroquine or chloroquine anti-HIV-1 activity was demonstrated in conjunction with antiretroviral drugs such as zidovudine, hydroxyurea, and didanosine in vitro, or patients (Paton and Aboulhab 2005).

Hydroxychloroquine raises the $\mathrm{pH}$ in intracellular vacuoles and improves processes such as protein degradation by acidic lysosome hydrolases, assembly of macromolecules in endosomes, and post-translation protein modification in the Golgi apparatus. It is suggested that the antirheumatic properties of these compounds derive from their involvement in macrophages and other antigen-presenting cells with the "antigen processing". These compounds give greater confidence in aspects of the clinical and laboratory parameters. These are distinguished from glucocorticoids and non-steroidal anti-inflammatory agents by their slow start of the action.

\section{Conclusion}

This latest virus epidemic has challenged most countries across the globe with economic, medical, and public health infrastructures. Time alone can tell how the virus is going to affect our lives. Potential outbreaks of the viruses and pathogens of zoonotic origin are likely to occur. Hydroxychloroquine has been used for 70 years as a cheap and safe drug, and is, thus, potentially clinically important against COVID-19. There is hope in this high-need scenario that chloroquine could change the whole history of this pandemic assault.

\section{Compliance with ethical standards}

Conflict of interest The authors have no competing interests to declare.

Ethical approval and informed consent Not applicable.

\section{References}

Bauch CT, Lloyd-Smith JO, Coffee MP, Galvani AP (2005) Dynamically modeling SARS and other newly emerging respiratory illnesses: past, present, and future. Epidemiology 16(6):791-801

Byrd TF, Horwitz MA (1991) Chloroquine inhibits the intracellular multiplication of Legionella pneumophila by limiting the availability of iron. A potential new mechanism for the therapeutic effect of chloroquine against intracellular pathogens. J Clin Invest 88:351-357

Cascella M, Rajnik M, Cuomo A, Dulebohn SC, Di Napoli R (2020) Features, evaluation and treatment coronavirus (COVID-19). In: StatPearls [Internet]. StatPearls Publishing, Treasure Island, FL. Available from: https://www.ncbi.nlm.nih.gov/books/NBK554776/

Centers for Disease Control and Prevention (CDC) (2020) Information for clinicians on investigational therapeutics for patients with COVID-19. https://www.cdc.gov/coronavirus/2019-ncov/hcp/ therapeutic-options.html\#r1. Accessed: 24 Mar 2020

Chen Y, Liu Q, Guo D (2020) Emerging coronaviruses: genome structure, replication, and pathogenesis. J Med Virol 92:418-423. https://doi. org/10.1002/jmv.25681

Huang C, Wang Y, Li X et al (2020) Clinical features of patients infected with 2019 novel coronavirus in Wuhan, China. Lancet 395:497506. https://doi.org/10.1016/S0140-6736(20)30183-5

Hui DS, Azhar EI, Madani TA et al (2020) The continuing 2019-nCoV epidemic threat of novel coronaviruses to global health - the latest 2019 novel coronavirus outbreak in Wuhan, China. Int J Infect Dis 91:264-266

Ji W, Wang W, Zhao X, Zai J, Li X (2020) Cross-species transmission of the newly identified coronavirus 2019-nCoV. J Med Virol 92:433440

Kupferschmidt K, Cohen J (2020) WHO launches global megatrial of the four most promising coronavirus treatments. Available from: http:// www.sciencemag.org/news/2020/03/who-launches-globalmegatrial-four-most-promising-coronavirus-treatments

Li Q, Guan X, Wu P et al (2020) Early transmission dynamics in Wuhan, China, of novel coronavirus-infected pneumonia. N Engl J Med 382(13):1199-1207. https://doi.org/10.1056/NEJMoa2001316

Mulangu S, Dodd LE, Davey RT Jr et al (2019) A randomized, controlled trial of Ebola virus disease therapeutics. N Engl J Med 381:22932303. https://doi.org/10.1056/NEJMoa1910993 
Paton NI, Aboulhab J (2005) Hydroxychloroquine, hydroxyurea and didanosine as initial therapy for HIV-infected patients with low viral load: safety, efficacy and resistance profile after 144 weeks. HIV Med 6(1):13-20

Savarino A, Boelaert JR, Cassone A, Majori G, Cauda R (2003) Effects of chloroquine on viral infections: an old drug against today's diseases. Lancet Infect Dis 3(11):722-727

Su S, Wong G, Shi W et al (2016) Epidemiology, genetic recombination, and pathogenesis of coronaviruses. Trend Microbiol 24:490-502. https://doi.org/10.1016/j.tim.2016.03.003

Vincent MJ, Bergeron E, Benjannet S et al (2005) Chloroquine is a potent inhibitor of SARS coronavirus infection and spread. Virol J 2:69. https://doi.org/10.1186/1743-422X-2-69

Wang M, Cao R, Zhang L et al (2020) Remdesivir and chloroquine effectively inhibit the recently emerged novel coronavirus (2019nCoV) in vitro. Cell Res 30(3):269-271. https://doi.org/10.1038/ s41422-020-0282-0
Warren TK, Jordan R, Lo MK et al (2016) Therapeutic efficacy of the small molecule GS-5734 against Ebola virus in rhesus monkeys. Nature 531:381-385. https://doi.org/10.1038/nature17180

World Health Organization (WHO) (2020) Coronavirus disease (COVID-2019) situation reports. Situation report - 71, 31 March 2020. Available from: https://www.who.int/emergencies/ diseases/novel-coronavirus-2019/situation-reports. Accessed: 1 Apr 2020

Wu F, Zhao S, Yu B et al (2020) A new coronavirus associated with human respiratory disease in China. Nature 579:265-269. https:// doi.org/10.1038/s41586-020-2008-3

Zhou P, Yang X-L, Wang X-G et al (2020) A pneumonia outbreak associated with a new coronavirus of probable bat origin. Nature 579: 270-273. https://doi.org/10.1038/s41586-020-2012-7

Publisher's note Springer Nature remains neutral with regard to jurisdictional claims in published maps and institutional affiliations. 